\title{
Prescription, OTC and Complementary Medicine Use among Hmong Adults in Central California
}

Felicia Hodge ${ }^{\star}$, Suzanne Kotkin-Jaszi, Mohammad Rahman and Fernando Martinez

University of California at Los Angeles, Nursing, Los Angeles, CA 90095-1702, United States

*Corresponding author: Felicia Hodge, Professor, University of California at Los Angeles, Nursing, 700 Tiverton, Rm 5-940 Factor Bldg, Los Angeles, CA 90095-1702, United States, Tel: 310-210-9887; E-mail: feliciash@aol.com,

Received date: May 13, 2015; Accepted date: June 23, 2015; Published date: July 1, 2015

Copyright: (c) 2015 Felicia Hodge et al. This is an open-access article distributed under the terms of the Creative Commons Attribution License, which permits unrestricted use, distribution, and reproduction in any medium, provided the original author and source are credited.

\section{Introduction}

Prescription medication and non-prescription medication (otherwise known as over-the-counter, or OTC medication) are common and readily available to treat illness. Many individuals, however, choose the of use complementary medicine instead of or in conjunction with prescription or OTC medication. The reasons for using complementary medicines and its perceived effectiveness may vary among age, gender, or racial/ethnic groups. Better understanding of health-seeking behaviours, particularly in regards to medication use, can aid healthcare providers in counselling and treating indigenous populations such as the Hmong.

Studies document that the Hmong continue to prefer their own ancient medical traditions even after they have immigrated to the United States $[1,2]$. The use of shamanistic ceremonies in combination with Western biomedical medicine is preferred by elder Hmong Americans and their family caregivers [3]. Little is understood concerning the use of complementary and alternative medicine (CAM) but recent meta-analysis of studies of the Hmong found that CAM may not be an alternative practice, but rather the dominant form in their home countries [4,5]. The combined use of Western medicines and complementary Hmong medicines, and the identity of the condition that each is used for is of interest to researchers and providers alike as mixed medication may be harmful and providers require full disclosure of medication for appropriate medication counselling.

The Hmong are an indigenous group originating in the mountains and hills in Northern Laos. Since the end of the Vietnam War in the 1970s, groups of Hmong families fled their traditional homes in the mountains of North-eastern Laos or face persecution or death from the communist Pathet Lao. The Central Valley was a popular choice for those who came to California, because of the availability of agricultural land that allowed these refuges to continue their farming tradition. Because the Hmong has been exposed to Western medicine since the mid1900s, the use of dual medication use is becoming more frequent. Complementary herbal medicine, however, continues to be used to treat various illness and physical symptoms.

Complementary medicine is comprised of herbal remedies, meditation, visualization, message and chiropractic care, spiritual practices and prayer, acupuncture and vitamins. The Hmong of the Laos Highlands traditionally use herbs and spirituality to heal. Depending upon the group, categories of complementary medicine use may be used exclusively, in combination, or in prescribed stages. Also, the use of complementary medicine can be exclusive or in conjunction with prescribed or OTC medication.
The use of prescription, OTC, and complementary medicine use among a community of Hmong in Central California is the focus this paper. We identify the condition(s), related communications, and the perceived benefits of various medications. Recommendations for treatment and counselling indigenous groups are provided.

\section{Methods}

\section{Sample}

One hundred and seven adult Hmong participated in the study; 58 (54\%) males and 49 (46\%) females. Inclusion criteria included: (1) age 18 years and older, (2) self-identified Hmong, (3) resident of the city of Fresno, California, and (4) member of the selected household.

\section{Design}

A cross-sectional household study was implemented among the Hmong residents of Fresno, CA. To obtain the recruitment frame of Hmong households, the Fresno-Clovis, California telephone book was used to identify Hmong surnames. Undergraduate students, under the supervision of a faculty member, called the 17 telephone numbers associated with Hmong surnames and asked if they would be willing to participate in a 15-30 minute survey. All participated and the total number of adults recruited from the 17 households was 107, or approximately 6-7 adults per household. Arrangements were made to administer the survey in person to all adult Hmong members of the selected household. Interpreters were provided for those who could not speak English or who requested an interpreter.

\section{Data collection method}

A questionnaire on health practices and use of prescription drugs and complementary medicines was administered in a structured approach. Trained undergraduate students from the Fresno State University Department of Public Health contacted each identified household by telephone, US mail and by personal contact to recruit respondents and to coordinate the student-administered survey with each adult resident of the household. Respondents were provided verbal and written information on the study and consenting took place in their households or in the clinic/community meeting room. The voluntary nature of the study was explained, carefully noting that services at health care clinics or community centres were not contingent upon their participation in the study. Institutional Review Board (IRB) approval was obtained from Fresno State University, Department of Public Health, and Committee for the Protection of Human Subjects. Surveys were collected and hand-carried to the 
Page 2 of 5

project leader, who then coded each complete survey to protect confidentiality as individual identifies such as names, addresses were not recorded.

The following items were measured in the survey:

Socio-demographics: Questions were asked regarding participant's age, gender, marital status, education, and employment status. In addition, questions were asked if they needed an interpreter to assist them in their conversation with providers and their ability to read instruction labels.

Health-seeking behaviours: Participants were asked if they seek the help of healthcare providers or shamans for their illness. They were also asked when, why and how participants sought out health care.

Use of prescription, OTC, and complementary medicine: Reasons for use, importance of use, treatment effectiveness, and the last time used of the following: prescriptions ordered by physicians, over-thecounter medication, and complementary medicines. Each measure was dichotomized into two categories for the chi-square test.

Communication with provider: Participants were asked if they informed their provider about their use of prescription, OTC, and complementary medicines. They were also asked if their provider spent enough time with them, discussed medication side effects, if they asked the provider questions, and if they understood the medication instructions.

\section{Data analysis}

The completed surveys were cleaned and coded. The results were entered into an Excel computer program and analysed using the SAS Statistical Analysis Program (SAS Institute, 2011). Chi-square tests of association were used to assess if there was a relationship, or statistical difference in the measures. The study's outcome variable of interest, use of medication, was examined among the categories of prescription, OTC, and traditional medicine. Differences were further examined by importance of medicine/drug use, and the last time used. Health beliefs (usefulness and purpose of medication use by prescription, OTC and complementary medicine) were examined and healthseeking behaviours (communication with physician and outreach) was reported. All statistical analyses were performed with a Statistical Analysis Program (SAS/STAT) (Cary, NC, 2011). Statistical significance was set at $\mathrm{p}<0.05$.

\section{Findings}

Table 1 reports on the socio-demographic characteristics of the study participants. A little over one-half (54\%) of the 107 Hmong who participated in the study were male, $54 \%$ were married, $49 \%$ unemployed, and $26 \%$ had no formal education. Participant age ranged from 18-70 years. Forty-nine percent reported they used an interpreter because of language barriers, nevertheless, $77 \%$ reported that they were able to read the instructions on medication labels (Table $1)$.

\begin{tabular}{|l|l|}
\hline \multirow{2}{*}{ Demographic Characteristic } & Overall \\
\cline { 2 - 2 } & $\begin{array}{l}(\mathbf{N}=107) \\
\mathbf{n}(\%)\end{array}$ \\
\hline Gender & \multicolumn{2}{|l}{} \\
\hline Male & $58(54.2)$ \\
\hline
\end{tabular}

\begin{tabular}{|c|c|}
\hline Female & $49(45.8)$ \\
\hline \multicolumn{2}{|l|}{ Marital Status } \\
\hline Single & $36(33.6)$ \\
\hline Married & $58(54.2)$ \\
\hline Divorced & $7(6.5)$ \\
\hline Widow & $6(5.6)$ \\
\hline Widower & 0 \\
\hline NA & 0 \\
\hline \multicolumn{2}{|c|}{ Highest level of education } \\
\hline No Education & $28(26.1)$ \\
\hline Less than HS & $2(1.8)$ \\
\hline HS & $32(29.9)$ \\
\hline College & $40(37.3)$ \\
\hline Other & $3(2.8)$ \\
\hline NA & $1(0.9)$ \\
\hline \multicolumn{2}{|c|}{ Employment status } \\
\hline Part time & $11(10.2)$ \\
\hline Full time & $41(38.3)$ \\
\hline Unemployed & $52(48.6)$ \\
\hline Retired & $2(1.8)$ \\
\hline Other & 0 \\
\hline NA & $1(0.9)$ \\
\hline \multicolumn{2}{|c|}{ Need interpreter } \\
\hline Yes & $52(48.6)$ \\
\hline No & $55(51.4)$ \\
\hline \multicolumn{2}{|c|}{ Able to read instruction label } \\
\hline Yes & $82(76.6)$ \\
\hline No & $25(23.3)$ \\
\hline
\end{tabular}

Table 1: Socio-demographic characteristics of adult Hmong participants $(\mathrm{N}=107)$.

The health beliefs of respondents were measured by individual's perceived usefulness and purpose of medication/drug use. More respondents reported that prescription medication were more helpful (77.6\%) than OTC (67.3\%) or complementary medication (43.0\%). In addition, $61 \%$ of the sample reported that prescription drugs treated most of their symptoms, as compared to $56 \%$ for OTC and $47 \%$ complementary medicine. Females were significantly more likely to report that they used complementary medicines within the past year $(39.5 \%, p=0.025)$. This finding was significantly higher than the small percentage reported by males (14\%). When asked "How important is it for you to use" these drugs respondents were more favourable in their responses as $72 \%$ felt that complementary medicine was somewhat, 
Page 3 of 5

important, or very important and $85 \%$ of prescription and OTC drug use was reported in these categories. Prescription and OTC drugs were used largely for pain $(66.7 \%$ and $72.9 \%$ respectively), whereas only $8.4 \%$ use complementary medicine for pain. Sixty-seven percent reported complementary medicine/drugs were used for such ailments as hypertension, stomach and intestinal problems, and other illnesses.

Health-seeking behaviours were observed in participant's choice of healthcare provider $(61.7 \%)$ over OTC drugs or shamans/herbal medicines $(21.4 \%)$ for illness or injury. However, $51.4 \%$ reported that they turn to complementary medicine if they cannot pay for healthcare from western providers. Seventy-five percent felt that the shaman was helpful, $83 \%$ felt that it is important for them to ask a shaman to treat their illness, and $41.1 \%$ used a shaman within the past year.

\section{Use of Prescription Medication}

Sixty-two percent of the participants reported that they seek the help of healthcare providers for various illnesses, and that these encounters result in the use of prescription medication. Fifty-five percent used prescription medications during the past year for several physical complaints, largely for pain in the chest, neck, or back (66.6\%) or for intestinal illness such as diarrhoea, hypertension (high blood pressure), and other illness (33.3\%). Because of language barriers (the majority of participants either did not speak English or identified English as a second language) $48.6 \%$ needed an interpreter to assist them in their conversation with providers. A major barrier to obtaining prescription medication was financial; $51.4 \%$ reported they had problems paying for their prescription medication.

When we examined medication use by gender, differences were observed between those who reported prescription drug use, over-thecounter use, and complementary medicine (Table 2). Fifty percent of Hmong males reported using prescription drugs over their lifetime, a little more than females $(51.8 \%$ males v. $48.2 \%$ females). Males consistently reported higher prescription use for pain symptoms (23.9\% v. $18.2 \%)$, and males and females equally felt it' use was more important $(42.9 \%$ v. $42.9 \%)$. Both males and females reported that prescription drugs were very helpful/a lot helpful $(46.2 \%$ v. $45.2 \%)$ to them. Table 3 reports on the use of prescription, OTC, and complimentary medication use among the participants. A chi-square analysis shows statistical significance between all three categories for each of the questions. This table shows that although participants used prescription drugs, they continued to use it in conjunction with OTC and CAM. Also, use of prescription medication was used by 55\% within the past year, and it was used for chest, back, and neck pain $(67 \%)$, and it was deemed as very helpful (85\%). A majority of participants reported that the prescription drugs were positive and helpful (88\%) and very few (13\%) thought it was negative, dangerous, or evil.

\begin{tabular}{|l|l|}
\hline COMMUNICATION & $\mathbf{N}(\%)$ \\
\hline $\begin{array}{l}\text { Did your health care provider talk to you } \\
\text { regarding the usage of your medication } \\
\text { after he gave you the prescription }\end{array}$ & $90(84.1)$ \\
\hline Yes & $17(15.8)$ \\
\hline No & $\begin{array}{l}\text { Did you feel your health care provider } \\
\text { spend enough time explaining the } \\
\text { usage of your medication }\end{array}$ \\
\hline
\end{tabular}

\begin{tabular}{|c|c|}
\hline Yes & 88 (82.2) \\
\hline No & $19(17.7)$ \\
\hline \multicolumn{2}{|l|}{$\begin{array}{l}\text { Did your health care provider talk about } \\
\text { the side effects of your medications }\end{array}$} \\
\hline Yes & 77 (71.9) \\
\hline No & $30(28.0)$ \\
\hline \multicolumn{2}{|l|}{$\begin{array}{l}\text { Did your health care provider talk to you } \\
\text { about the possible interaction of using } \\
\text { multiple medication at once }\end{array}$} \\
\hline Yes & $75(70.1)$ \\
\hline No & $32(29.9)$ \\
\hline \multicolumn{2}{|l|}{$\begin{array}{l}\text { Did the clinic you visit have an } \\
\text { interpreter }\end{array}$} \\
\hline Yes & $76(71.0)$ \\
\hline No & $31(28.9)$ \\
\hline \multicolumn{2}{|l|}{$\begin{array}{l}\text { Did you need an interpreter to assist } \\
\text { your conversation with your health care } \\
\text { provider }\end{array}$} \\
\hline Yes & $52(48.6)$ \\
\hline No & 55 (51.4) \\
\hline
\end{tabular}

Table 2: Communications between Hmong patients and providers $(\mathrm{N}=107)$.

\section{Use of OTC Medication}

Study participants indicated that over-the-counter (OTC) medications were used frequently and often in conjunction with prescription medication. Sixty-one percent of participants reported that they used OTC medication within the past year. There was little or no difference in the OTC use patterns between male and female participants. Eighty-two percent of participants $(46.7 \%$ male and $35.6 \%$ female) used OTC medications for bodily complaints such as stomach and intestinal complaints, hypertension, asthma and other illness symptoms. Few used OTC for head and body pain (7.5\% male, $11.2 \%$ female). Ninety-three percent felt that OTC drugs were very helpful to treat their physical complaints, and $74 \%$ felt it as positive (Table 3), $25 \%$ felt the use of OTC medication it was "necessary but evil." 
Citation: Hodge F, Kotkin-Jaszi S, Rahman M, Martinez F (2015) Prescription, OTC and Complementary Medicine Use among Hmong Adults in Central California . J Nurs Care 4: 276. doi:10.4172/2167-1168.1000276

Page 4 of 5

\begin{tabular}{|c|c|c|c|c|}
\hline Drug Use & $\begin{array}{l}\text { Prescript-ion drug } \\
\text { use } \\
\mathrm{N}(\%)\end{array}$ & $\begin{array}{l}\text { O-T-C } \\
\text { drug use } \\
\mathrm{N}(\%)\end{array}$ & $\begin{array}{l}\text { Complimentary drug use } \\
\mathrm{N}(\%)\end{array}$ & p-value \\
\hline \multicolumn{5}{|c|}{$\begin{array}{l}\text { When was the last time you took prescription/OTC/ } \\
\text { complementary-herbal drugs? }\end{array}$} \\
\hline Less than 1 year ago & $47(54.65)$ & $49(74.24)$ & $29(72.50)$ & 0.0232 \\
\hline More than 1 year & $39(45.35)$ & $17(25.76)$ & $11(27.50)$ & \\
\hline \multicolumn{5}{|c|}{ Why did you use prescription/OTC/ complementary drugs? } \\
\hline Chest/back/neck pain & $38(66.67)$ & $35(72.92)$ & $10(33.33)$ & $0.0013^{*}$ \\
\hline Hypertension/stomach/intestinal/other illness & $19(33.33)$ & $13(27.08)$ & $20(66.67)$ & \\
\hline \multicolumn{5}{|c|}{$\begin{array}{l}\text { Did prescription/OTC/ complementary drugs treat most of your } \\
\text { symptom? }\end{array}$} \\
\hline \multicolumn{5}{|l|}{ Does not help at all } \\
\hline \multirow[t]{2}{*}{ Very helpful/helps a lot } & $12(15.38)$ & $7(6.60)$ & $31(29.52)$ & $0.0001^{*}$ \\
\hline & $66(84.62)$ & $99(93.40)$ & $74(70.48)$ & \\
\hline \multicolumn{5}{|c|}{$\begin{array}{l}\text { How important for you to use prescription/OTC/ complementary } \\
\text { drugs? }\end{array}$} \\
\hline Not/modest important & $14(13.33)$ & $12(11.32)$ & $28(26.67)$ & $0.0057^{*}$ \\
\hline Somewhat/very important & $91(86.67)$ & $94(88.68)$ & $77(73.33)$ & \\
\hline \multicolumn{5}{|c|}{ Prescription/OTC/ complementary drugs are something } \\
\hline Positive/helpful & $83(87.37)$ & $72(74.23)$ & $46(52.87)$ & $0.0001^{*}$ \\
\hline Negative/danger/ evil & $12(12.63)$ & $25(25.77)$ & $41(47.13)$ & \\
\hline
\end{tabular}

Table 3: Use of prescription, OTC, and complimentary medication use.

\section{Use of Traditional Medication}

As reported above, significantly more females than males reported using complementary medicines during the past year (39.5\% female vs. $14 \%$ male, $\mathrm{p}=0.025$ ). Bodily symptoms, such as stomach and intestinal problems, asthma, and other symptoms were reasons for the use of herbs and other traditional medicines. Both males and females $(34.3 \%$, $35.2 \%$ respectively) thought that herbal medicines treated most of their symptoms and that it was very important medicine (50\% male vs. $43.3 \%$ female).

Seeking the services of a shaman for physical complaints was reported by a large percentage of participants (75.7\%). Participants explained that they felt going to a shaman or a spiritual healer was a positive event (65\%), and that the treatments were helpful $(20.6 \%)$, very helpful (34.6\%), or helps a lot (38.3\%). Only $10.3 \%$ felt going to a shaman was a "necessary but evil" event.

\section{Communication}

Although $49 \%$ of Hmong reported that they needed an interpreter to assist in conversation with their doctor (as $71 \%$ of the clinics they visited had an interpreter available to them), a large percent (82.2\%) of the respondents reported that they felt their health care provider spent enough time explaining the usage of their prescribed medication. Eighty-four percent reported that the provider talked about the usage of the prescription, possible interaction of using multiple medications at once $(70.1 \%)$, and medication side effects $(71.9 \%)$.

\section{Discussion}

This study showed that while seeking assistance from health care providers is the preferred choice of many Hmong residents in Central California (62\%), a significant number $(75.7 \%)$ continued to use complementary medicine (from a shaman) to treat illness. These study results find a high percentage of Hmong residents in the Central Valley utilizing complementary medicine were also reported in a study by Helsel et al. in 2004 in Merced, California, a community in the Central Valley with a population of over 7,000 Hmong Americans. Similarly, an earlier study in the Central Valley conducted by Nutall and Flores in 1997 found that the Hmong are more likely to use shamanistic practices, herbal remedies, or talismans rather than western biomedical practices [6]. This is especially true for problems related to soul loss and loss of life visa [7]. Over-the-counter medications were used primarily for bodily symptoms and prescription medication was sought out for pain primarily of the chest, neck and head.

Programs targeting Hmong patients should be aware of the high usage of complementary medicine, indeed the possible co-mingling of prescription, OTC and herbal medicines for bodily symptoms. Culturally appropriate education should be employed to improve and 
Page 5 of 5

maintain communication and healthcare interactions. It is also important to recognize that it has been almost thirty years since the first Hmong immigrants began to arrive in the US. For younger Hmong who were born in the US, there are many different health care options available from traditional Hmong shamanic and herbal practices to any number of complementary medical practices or to seeing specialized health care clinics with Hmong American physicians and nurse practitioners. Hmong Americans come from a culture that focuses on the group and family rather than the individual. By seeking out a shaman and utilizing traditional herbal medicines in combination with western biomedical practices, it may help to restore a sense of cultural connection to their group, their homeland, and those who were left behind because they had passed away or were too elderly to immigrate to the United States. Health care workers including nurses, public health educators and administrators need to be culturally sensitive to these traditional practices and recognize they may provide a deep sense of comfort and solace during an episode of illness. Suffering an illness while seeking care in a complex health care system, the Hmong are also often burdened by the cost of care as almost half of the respondents reported being unemployed. This stressful interaction with the western health care system is also exacerbated by the language barrier. It is important to recognize that as long as the herbal medications and shamanic practices are not harmful, they are not competing with western medicine but supporting the patient in a time that most patients can find stressful.

\section{Conclusion}

For nurses and public health workers who practice in areas with significant populations of Hmong Americans including California's Central Valley, Minnesota, Wisconsin, North Carolina and other areas of refugee resettlement, it is of importance to understand the Hmong culture and historical experience. It is recommended that targeted healthcare practices that are culturally tailored to include both western biomedical and traditional herbal Hmong healing practices to show the greatest promise for providing Hmong Americans with high quality, culturally appropriate health care.
Mary Jo Berghtol, who worked as a nurse in Fresno, California explained the confusion Hmong refugees face in understanding the differences between traditional Hmong medical practices and the complex US health care system said, "They have jumped 2,000 years in a matter of a few days." The magnitude of this cultural transition is difficult for non-Hmong health care workers to grasp and address. This paper seeks to explain how these extremely divergent medical practices impact use of OTCs, traditional medicines and western pharmaceuticals.

The transcultural model of nursing suggests that respect of the patient's own culture is critical for all patients with the quality care that the US health care system can deliver. As a pioneering nurse theorist, Madeline Leninger recognized that lack of knowledge of culture was a "missing link" for nurses to understand how to tailor patient care to increase adherence, healing and patient wellness [8].

\section{References}

1. Gerdner L (2012) Shamanism: indications and use by older Hmong Americans with chronic illness. Hmong Studies Journal, 13: 1-22.

2. Thao X (1986) Hmong perceptions of illness and traditional ways of healing. In: The Hmong in transition, Hendricks G, Downing B, Deinard A (eds.), Center for Migration Studies, New York.

3. Cha D (2005) Hmong American Concepts of Health, Healing, and Conventional Medicine, Taylor and Francis, New York.

4. MacDuff S, Grodin MA, Gardiner P (2011) The use of complementary and alternative medicine among refugees: a systematic review. J Immigr Minor Health 13: 585-599.

5. Gerdner L (2011) Health and health care of Hmong American older adults In: VS Periyakoil (ed.), eCampus Geriatrics, Stanford, CA.

6. Nuttall P, Flores FC (1997) Hmong healing practices used for common childhood illnesses. Pediatr Nurs 23: 247-251.

7. Helsel DG, Mochel M, Bauer R (2004) Shamans in a Hmong American community. J Altern Complement Med 10: 933-938.

8. Leninger M Madeline Leniger's cultural care: diversity and universality theory. 